\title{
Identification of the Neuroprotective Molecular Region of Pigment Epithelium-Derived Factor and Its Binding Sites on Motor Neurons
}

\author{
Masako M. Bilak, ${ }^{1}$ S. Patricia Becerra, ${ }^{2}$ Andrea M. Vincent, ${ }^{3}$ Brian H. Moss, ${ }^{1}$ Maria S. Aymerich, ${ }^{2}$ and \\ Ralph W. Kuncl ${ }^{4}$ \\ ${ }^{1}$ Department of Neurology, Johns Hopkins University School of Medicine, Baltimore, Maryland 21287, ${ }^{2}$ National Eye \\ Institute, National Institutes of Health, Bethesda, Maryland 20892, 32Department of Neurology, University of Michigan, \\ Ann Arbor, Michigan 48109, and 4Department of Biology, Bryn Mawr College, Bryn Mawr, Pennsylvania 19010
}

Pigment epithelium-derived factor (PEDF), a member of the serine protease inhibitor (serpin) family, is a survival factor for various types of neurons. We studied the mechanisms by which human PEDF protects motor neurons from degeneration, with the goal of eventually conducting human clinical trials. We first searched for a molecular region of human PEDF essential to motor neuron protection. Using a spinal cord culture model of chronic glutamate toxicity, we show herein that a synthetic 44 mer peptide from an $\mathrm{N}$-terminal region of the human PEDF molecule that lacks the homologous serpin-reactive region contains its full neuroprotective activity. We also investigated the presence and distribution of PEDF receptors in the spinal cord. Using a fluoresceinated PEDF probe, we show that spinal motor neurons contain specific binding sites for PEDF. Kinetics analyses using a radiolabeled PEDF probe demonstrate that purified rat motor neurons contain a single class of saturable and specific binding sites. This study indicates that a small peptide fragment of the human PEDF molecule could be engineered to contain all of its motor neuron protective activity, and that the neuroprotective action is likely to be mediated directly on motor neurons via a single class of PEDF receptors. The data support the pharmacotherapeutic potential of PEDF as a neuroprotectant in human motor neuron degeneration.

Key words: amyotrophic lateral sclerosis; motor neurons; neurodegeneration; neuroprotection; neurotrophic factor; peptide; pigment epithelium-derived factor; serpin; spinal cord
Pigment epithelium-derived factor (PEDF) was initially identified as a potent neurotrophic factor for retinal neurons. In the eye, PEDF increases the survival and differentiation of Y-79 retinoblastoma cells (Becerra, 1997), has morphogenetic effects on the development of photoreceptors of Xenopus laevis (Jablonski et al., 2000), delays the degeneration of photoreceptors in animal models of inherited retinitis pigmentosa (Cayouette et al., 1999) and of light-induced damage (Cao et al., 2001), and inhibits the proliferation of endothelial cells and angiogenesis (Dawson et al., 1999; Gao et al., 2001; Mori et al., 2001; Ogata et al., 2001; Stellmach et al., 2001). PEDF has neurotrophic and neuroprotective functions in the CNS beyond the retina. For example, PEDF supports the survival of immature cerebellar granule (CG) neurons (Taniwaki et al., 1995) and protects them from induced apoptosis (Araki et al., 1998; Nomura et al., 2001), protects older CG neurons from glutamate injury (Taniwaki et al., 1997), and protects embryonic hippocampal neurons from glutamate injury (DeCoster et al., 1999). We and others have shown that fulllength and properly folded native PEDF protects postnatal rat motor neurons from chronic glutamate injury and embryonic

\footnotetext{
Received May 14, 2002; revised Aug. 14, 2002; accepted Aug. 16, 2002.

This work was supported by research grants from the National Institute of Neurological Disorders and Stroke and the Muscular Dystrophy Association, the Dino and Wendy Fabbri Fund, the Sue Mullen Fund, the Mid Atlantic Fall Classic Fund, and the Gail Rupertus Fund for Neuromuscular Research. We thank Kati Andreasson for critical reading of this manuscript, Eva Feldman for providing lab facilities and reading this manuscript, Stephan Bilak for assistance in radioligand binding and neuroprotection experiments, and Irina Shats for general technical help. Correspondence should be addressed to Dr. Ralph W. Kuncl, Department of Biology, Bryn Mawr College, Taylor Hall, First Floor, 101 North Merion Avenue, Bryn Mawr, PA 19010. E-mail: rkuncl@brynmawr.edu.

Copyright () 2002 Society for Neuroscience 0270-6474/02/229378-09\$15.00/0
}

chick motor neurons from apoptosis (Bilak et al., 1999a; Houenou et al., 1999).

Human PEDF is a $50 \mathrm{kDa}$ glycoprotein, and molecular sequence analyses indicate that it belongs to the serine protease inhibitor (serpin) supergene family (Steele et al., 1993). It has a globular conformation with a single protease-sensitive loop that contains a homologous serpin-reactive site toward its $\mathrm{C}$ terminal (Becerra et al., 1995). Previous studies have implicated pathophysiologic roles in neurodegenerative diseases for certain serine proteases and serpins, e.g., thrombin, urokinase, and their inhibitors protease nexin-I and plasminogen activator inhibitor, respectively (Wagner et al., 1989; Festoff et al., 1996). However, no inhibitory activity has been reported for PEDF; therefore, it belongs to the noninhibitory subclass of serpins that includes angiotensinogen and ovalbumin (Doolittle, 1983; Becerra et al., 1993). Structure-function studies have shown that PEDF lacking the homologous serpin-reactive loop retains its neuronal differentiation, survival, and anti-angiogenic activities (Becerra et al., 1995; Araki et al., 1998; Dawson et al., 1999). A peptide of 44 aa from positions $78-121$ of the 418 aa human PEDF can induce neuronal differentiation on retinoblastoma cells. A truncated form of PEDF that lacks $\sim 62 \%$ of the carboxyl end of the polypeptide comprising the homologous serpin-reactive loop also promotes the survival and differentiation (neurite outgrowth) of embryonic chick spinal motor neurons (Houenou et al., 1999). Thus, the neurotrophic/neuroprotective activities of PEDF on neurons must be mediated via a mechanism that is independent of the inhibition of serine proteases.

Previous studies have shown evidence for the presence of a PEDF binding protein of $\sim 80 \mathrm{kDa}$ with characteristics of a PEDF receptor in retinoblastoma cells and CG cells (Alberdi et al., 
1999). It is likely that this receptor triggers the necessary signals for the resulting neurotrophic activity. However, the distribution and kinetics of PEDF receptors in the spinal cord have not yet been reported. Therefore, we set out to explore the mechanisms of action by which PEDF protects motor neurons from degeneration. We used synthetic peptides derived from the human PEDF sequence, the 34 mer (positions 44-77) and 44 mer (positions 78-121), to search for a molecular region of PEDF essential to motor neuron protection. We used fluorescein (Fl)-conjugated PEDF to investigate the presence and distribution of PEDF receptors on motor neurons. To determine the binding characteristics of PEDF receptors, we used radiolabeled PEDF in classical radioligand binding assays in spinal cord and isolated motor neuron cultures. Together, our data present compelling evidence for mechanisms of action independent of serine protease inhibition but involving the 44 mer region of PEDF in binding to receptors on the cell surface of the motor neuron in the spinal cord.

\section{MATERIALS AND METHODS}

Preparation of PEDF polypeptides. Recombinant human (rhu) PEDF was synthesized by baby hamster kidney cells containing the expression vector pMA-PEDF with a full-length human PEDF cDNA, and the recombinant PEDF protein was purified from the conditioned media (Stratikos et al., 1996). Two synthetic peptides, the 34 mer and the 44 mer, were designed from amino acid positions 44-77 (DPFFKVPVNKLAAAVSNFGYDLYRVRSSMSPTTN) and 78-121 (VLLSPLSVATALSALSLGAEQRTESIIHRALYYDLISSPDIHGT) of the human PEDF sequence (GenBank accession number U29953), respectively, and prepared by Biosynthesis, Inc. (Lewisville, TX), followed by HPLC purification ( $>90 \%$ purity) and N-terminal determination (Alberdi et al., 1999). The resulting peptides were soluble in aqueous solutions. rhuPEDF was commercially labeled with ${ }^{125}$ I (Lofstrand Labs Limited, Gaithersburg, MD), as described previously (Alberdi et al., 1999). The specific activity varied between 0.572 and $3.71 \times 10^{7} \mathrm{cpm} / \mu \mathrm{g}$, and the concentration varied between 0.371 and $1.80 \times 10^{6} \mathrm{cpm} / \mu \mathrm{l}$. rhuPEDF was chemically coupled with Fl-5-EX succinimidyl ester (Molecular Probes, Eugene, OR), as described previously (Aymerich et al., 2001a). Both ${ }^{125}$ I-PEDF and Fl-PEDF are biologically active, because they can induce neuronal differentiation in human retinoblastoma cells and promote survival of CG cell neurons (Alberdi et al., 1999; Aymerich et al., 2001b). The Fl-PEDF lacks binding affinity for glycosaminoglycans but has affinity for the PEDF receptor, making it suitable for receptor distribution studies (Aymerich et al., 2001a).

Neuroprotection paradigm. We tested the full-length molecule rhuPEDF and the peptides 44 mer and 34 mer for their neuroprotective efficacy using an in vitro organotypic spinal cord model of chronic glutamate-mediated motor neuron degeneration (Corse et al., 1999). The neuroprotective paradigm began with the addition of $\beta$-threohydroxyaspartate (THA) and rhuPEDF at $8 \mathrm{~d}$ in vitro (DIV), as we have described previously (Bilak et al., 1999a,b, 2001; Corse et al., 1999). In this culture model, we have shown that the addition of $100 \mu \mathrm{M}$ THA injures motor neurons with a morphology typical of excitotoxic degeneration, resulting in reduced total choline acetyltransferase (ChAT) activity over weeks, and causing depletion of ChAT- and SMI (Sternberger Monoclonals, Inc.)-32-immunopositive motor neurons (Rothstein et al., 1993; Bilak et al., 1999a,b, 2001; Corse et al., 1999). We examined three doses of PEDF protein and peptides $(0.04,0.4$, and $4 \mathrm{nM})$. Cultures were harvested or fixed after DIV 42-49, and motor neuron survival was assessed by ChAT radiochemical assays and motor neuron counts (Bilak et al., 1999a,b, 2001). We included untreated and THA-treated controls in each experiment. To determine the number of surviving motor neurons, fixed slice cultures were processed for immunohistochemistry for neurofilament-H (NF-H) with SMI-32 antibody (Sternberger Monoclonals, Lutherville, MD) as described previously (Bilak et al., 1999a,b). Motor neurons were counted by a single investigator on masked slides. Overall culture morphology was monitored using phase-contrast microscopy. To compare the effect of different rhuPEDF peptides, a neuroprotection index was calculated by subtracting the percentage of the relevant THA control within each treatment group from the average percentage of neuroprotection of each treatment group. At least triplicate wells for each experimental group were used in each experiment.

Neutralization of PEDF activity with biologically active anti-PEDF anti- body. To prove the specificity of the neuroprotective action of PEDF in our model, we tested whether a neutralizing rabbit antiserum antirhuPEDF can block its neuroprotective effect. This antiserum has been shown to block several biological activities of PEDF (Wu et al., 1995; Taniwaki et al., 1997). rhuPEDF (4 nM) was preincubated with the antiserum $(1: 600-1: 1200)$ at $4^{\circ} \mathrm{C}$ overnight before being added to the culture medium. PEDF alone, antiserum alone, or PEDF preadsorbed with antiserum were each added simultaneously with THA to the culture medium on DIV 8 and thereafter. Cultures were harvested after DIV 42; ChAT assay was the outcome measure. To determine whether the antibody had intrinsic (trophic or toxic) effects on untreated cultures or THA-treated cultures, some normal and THA-intoxicated control cultures were also incubated with the antibody alone.

Statistical analyses of cultures for neuroprotection. Statistical analysis of continuous data (ChAT activity) was performed by one-way ANOVA, followed by two-tailed Student's $t$ tests to compare the effects of each PEDF peptide with the THA-treated control. Identical treatment groups from multiple experiments were combined after normalization to the mean of THA controls within an individual experiment. All ChAT activity data are reported as means \pm SEM.

The quantitative analyses of counted motor neurons, considered nearordinal data, required the nonparametric Kruskal-Wallis ANOVA to analyze multiple tested peptides at multiple doses. This was followed by the Mann-Whitney $U$ test to compare each drug treatment with the THA control. Identical treatment groups from multiple experiments were combined after normalization to the mean of THA controls within an individual experiment. Motor neuron count data are illustrated in a "box-and-whisker" plot, in which the box represents 25th and 75th percentiles, the bar indicates the median, and the whiskers represent 5 th and 95 th percentiles.

Two-tailed analyses were always performed. We used $p<0.05$ as the level of significance.

Preparation of motor neuron cultures. Motor neuron-enriched cultures from embryonic day 16 Sprague Dawley rats were prepared as described previously (Houenou et al., 1999), with slight modifications. The purity of motor neurons was validated using islet-1 and SMI-32 as motor neuron markers (Ericson et al., 1992; Tsang et al., 2000). At least $80-85 \%$ of the cells prepared by this method are immunopositive for SMI-32 (1:1000) or islet-1 (1:100; Developmental Studies Hybridoma Bank, Iowa City, IA) (see Fig. 5). Cells were plated on a poly-L-lysine-coated $(0.05 \mathrm{mg} / \mathrm{ml}) 24$ well plate and maintained at $37^{\circ} \mathrm{C}$ for $2-3 \mathrm{hr}$ in Neurobasal-A-based "motor neuron medium" containing the following components: B27, albumin, catalase, superoxide dismutase (SOD), transferrin, galactose, progesterone, putrescine, selenium, $\beta$-estradiol, hydrocortisone, glutamine, and penicillin/streptomycin/neomycin $(\mathrm{P} / \mathrm{S} / \mathrm{N})$. All chemicals were purchased from Sigma (St. Louis, MO), with the exception of P/S/N and B27 (Invitrogen, Paisley, UK).

Biological responses of embryonic rat motor neurons to PEDF. It has been shown previously that PEDF enhances the survival of embryonic chick motor neurons (Houenou et al., 1999). We adapted that method to confirm that PEDF has a similar effect on our rat motor neuron preparation; we plated $\sim 20,000$ cells per well in the motor neuron medium described above and allowed them to attach to the substrate for $3 \mathrm{hr}$. Cells were then switched to a similar medium lacking B27 and glutamine, with or without $10 \mathrm{ng} / \mathrm{ml}$ PEDF. Positive control cultures were incubated in the motor neuron medium plus B27 for the same period of time. The medium was changed after $48 \mathrm{hr}$. At the end of the $96 \mathrm{hr}$ incubation period, the cells were fixed with $4 \%$ paraformaldehyde. We counted attached cells that had a morphology resembling motor neurons (i.e., containing at least one long neurite and a typically pyramidal- or bipolarshaped cell body) in five random fields $\left(\sim 1 \mathrm{~mm}^{2}\right)$ per each well using a phase-contrast microscope with a $20 \times$ objective lens; the total numbers of cells per well was calculated.

Fl-PEDF binding assays. To investigate the topographic distribution of the PEDF receptor in the rat spinal cord, slice cultures grown in Neurobasal-A medium/B27 for 1 week were removed from culture membrane inserts, transferred into 24 well tissue-culture plates, and incubated with $20 \mathrm{~nm}$ Fl-PEDF in "binding buffer" (Neurobasal-A plus $0.2 \%$ BSA) for $1-4 \mathrm{hr}$ at $4^{\circ} \mathrm{C}$. After a $30 \mathrm{~min}$ rinse with the binding buffer, cultures were either mounted for direct fluorescence imaging or subsequently processed for double-fluorescence with SMI-32 and Texas Redconjugated anti-mouse IgG (Rockland Immunochemicals, Gilbertsville, PA) to identify motor neurons in the ventral horn. A Zeiss (Thornwood, NY) Axiovert fluorescence microscope connected to IP Lab (Scanalytics, Fairfax, VA) Multicolor software was used for photography. We 
identified motor neurons by (1) size ( $>25 \mu \mathrm{m})$ and shape of the neuron, (2) location of the neuron in the ventral gray matter, and (3) immunoreactivity for NF-H (Bilak et al., 1999a,b). In some other cultures, a bound Fl-PEDF signal was enhanced by subsequent incubation with biotinylated anti-fluorescein mouse IgG (Rockland) and the VectaABC Elite Kit (Vector Laboratories, Burlingame, CA), and bound products were visualized with DAB (Fisher Scientific, Houston, TX). Controls were incubated with either Fl-PEDF in the presence of excess unlabeled PEDF or anti-fluorescein IgG-biotin in the absence of Fl-PEDF.

Fl-PEDF binding on motor neurons was performed by incubating a relatively pure population of motor neurons with Fl-PEDF, as follows. Motor neurons were prepared on coverslips in a 24-well tissue-culture plate, as described above, and grown for $2 \mathrm{hr}$. Cultures were rinsed with the binding buffer and chilled to $4^{\circ} \mathrm{C}$ for $30 \mathrm{~min}$. Fl-PEDF $(4,20$, or 50 nM) was added to the culture medium and incubated at $4^{\circ} \mathrm{C}$ for $2-12 \mathrm{hr}$. After a 30 min rinse with the binding buffer, cultures were fixed with $4 \%$ paraformaldehyde and coverslips were mounted for fluorescence imaging, as described above. Control wells were incubated with $20 \mathrm{~nm}$ FlPEDF in the presence of a 50 -fold excess of unlabeled PEDF.

Radiolabeled ${ }^{125}$ I-PEDF binding assays. PEDF binding assays on spinal cord slices were performed using ${ }^{125}$ I-labeled PEDF as radioligand. Spinal cord slices were grown in Neurobasal-A medium with B27 supplement (Invitrogen) for 1 week to allow stabilization after the axotomy of culture preparation. After a brief rinse with the binding buffer, cultures were incubated with ${ }^{125} \mathrm{I}-\mathrm{PEDF}(4 \mathrm{nM})$ for $0.25,1.5,4$, or $7.5 \mathrm{hr}$ at $4^{\circ} \mathrm{C}$ on DIV 9. After rinsing with cold binding buffer, cultures were removed from membrane inserts. Five slices were combined in a tube, and bound radioactivity was determined using a gamma counter. Nonspecific binding was determined by the incubation of cultures with ${ }^{125}$ I-PEDF (4 nM) in the presence of a 100 -fold excess of unlabeled PEDF. Specific binding was calculated by subtracting nonspecific binding from the total binding. All of the experimental points were given as the average of triplicates.

${ }^{125}$ I-PEDF binding to motor neurons was performed using cultures of $130,000-200,000$ motor neurons per well. Cells were plated on 24 well plates and grown for $3 \mathrm{hr}$. Before the binding analysis, the culture medium was removed and the motor neurons were incubated for $30 \mathrm{~min}$ with the binding buffer at $37^{\circ} \mathrm{C}$. One-half of this conditioned binding buffer was then pooled and used to prepare $2 \times{ }^{125}$ I ligand. Cells were chilled on ice for $15 \mathrm{~min}$. Radioligand $(2 \mathrm{nM})$ and increasing concentrations of unlabeled PEDF (0-200 nM) were added to the cells and incubated for $1 \mathrm{hr}$ at $4^{\circ} \mathrm{C}$. At the end of incubation, cells were rinsed three times with the binding buffer. Bound ligands in each well were removed after incubation with $0.2 \mathrm{ml} 1 \mathrm{~N} \mathrm{NaOH}$ for $30 \mathrm{~min}$ at room temperature and transferred to scintillation fluid for $\beta$-scintillation detection. Specific binding was determined by subtracting nonspecific binding (measured in the presence of a 100 -fold molar excess of unlabeled ligand) from the total binding. Data were analyzed with GraphPad Prism software (GraphPad Software Inc., San Diego, CA) to determine saturation curves by nonlinear regression and to perform Scatchard analyses for data display. Each experimental point corresponds to at least three replicates.

\section{RESULTS}

\section{The 44 mer peptide completely accounts for neuroprotection}

We compared the effect of the two PEDF peptides, 34 mer (spanning residue positions 44-77) and 44 mer (positions 78121 ), with that of the full-length rhuPEDF protein on the prevention of ChAT activity loss. Treatment with the 44 mer peptide at three doses $(0.04,0.4$, and $4 \mathrm{~nm})$ potently prevented the loss of spinal cord ChAT activity caused by chronic glutamate injury $(p<0.03$ by ANOVA) (Fig. $1 A, B)$. Full-length rhuPEDF protein was similarly effective in significantly preventing the loss of ChAT activity at corresponding doses (only 4 nm shown in Fig. $1 C$ ), as we have observed previously with native human PEDF (Bilak et al., 1999a). In contrast, the 34 mer peptide at the same three doses did not prevent the motor neuron ChAT activity loss (Fig. 1C). Neither rhuPEDF nor the peptides had any constitutive effect of their own on ChAT activity in otherwise untreated normal spinal cord slices (data not shown).
We also compared the effect of the two peptides, 34 mer and 44 mer, with that of the full-length rhuPEDF protein on motor neuron survival. Although THA toxicity was severe $(p=0.01$ vs untreated controls by Mann-Whitney $U$ test) (Fig. $1 D, G)$, the 44 mer $(0.04,0.4$, and $4 \mathrm{~nm})$ offered effective, dose-responsive neuroprotection ( $p=0.034$ by ANOVA) (Fig. 1E,H). When the neuroprotection index was examined, the 44 mer peptide (only 4 nM shown) was as highly protective as the rhuPEDF protein ( $p=$ 0.014 by ANOVA) (Fig. $1 F$ ). The 34 mer peptide at the same three doses did not provide neuroprotection ( $p=0.362$ by ANOVA) (Fig. 1F,I). These data combined indicate that the human PEDF region spanning amino acid positions 78-121 contains the structural determinant for protecting motor neurons in organotypic cultures from chronic glutamate injury.

\section{Neutralizing anti-PEDF antiserum blocks the neuroprotective activity of PEDF}

The specific antiserum against rhuPEDF has been shown to neutralize the neurotrophic activities of PEDF in retinoblastoma and CG cells (Wu et al., 1995; Taniwaki et al., 1997). To determine whether it could also neutralize the effect of PEDF on motor neurons, we preincubated PEDF with the antiserum and assayed for its activity. Preadsorption with antiserum at a dilution of 1:600 completely blocked the neuroprotective effect of $4 \mathrm{~nm}$ rhuPEDF (Fig. 2) ( $p=7.4 \times 10^{-4}$ vs "no antibody" group). Antiserum at a 1:1200 dilution provided a partial blockade of the activity of PEDF, indicating an antibody dose response. Cultures treated with PEDF antiserum alone (or plus THA) did not show any significant change in ChAT activity or gross morphology compared with appropriate controls, indicating that the observed effect was attributable to the blocking activity of the antiserum and not to any inherent toxic activity of the antiserum. These data suggest that the neuroprotective effect we observed is specific to PEDF.

\section{PEDF binding sites in spinal cord}

With Fl-PEDF, we observed a fluorescein signal on motor neurons in the gray matter of the ventral horn after a $1 \mathrm{hr}$ incubation with $20 \mathrm{~nm} \mathrm{Fl-PEDF} \mathrm{(Fig.} \mathrm{3A).} \mathrm{The} \mathrm{fluorescence} \mathrm{signal} \mathrm{appeared}$ in a punctate pattern on motor neurons after a $1 \mathrm{hr}$ incubation. After a much longer incubation (12 hr) or with a higher concentration of the Fl-PEDF (50 nM), the fluorescein signal was also consistently observed in the nucleus of motor neurons, which were identified with SMI-32 immunofluorescence staining (data not shown). This may be attributable to the uptake of Fl-PEDF or to its products of degradation by cells. Under identical settings, there was minimal amorphous background autofluorescence in untreated unlabeled spinal cord (Fig. 3B). Controls that were incubated for $1 \mathrm{hr}$ with $20 \mathrm{nM}$ Fl-PEDF in the presence of a 50-fold molar excess of unlabeled PEDF showed no binding above the background autofluorescence (data not shown). These observations imply that motor neurons in the spinal cord contain specific PEDF binding sites, and that the initial binding of PEDF occurs on the surface of the motor neurons.

\section{Localization of PEDF binding sites on purified rat motor neurons}

We performed experiments to localize PEDF binding sites in motor neurons in the same single-cell cultures as those used for the radioligand binding assays. Fl-PEDF was used to determine the distribution of PEDF binding sites on purified motor neurons that were grown on poly-L-lysine substrates. Virtually all SMI32-positive cells contained fluorescein signal in a punctate man- 
A
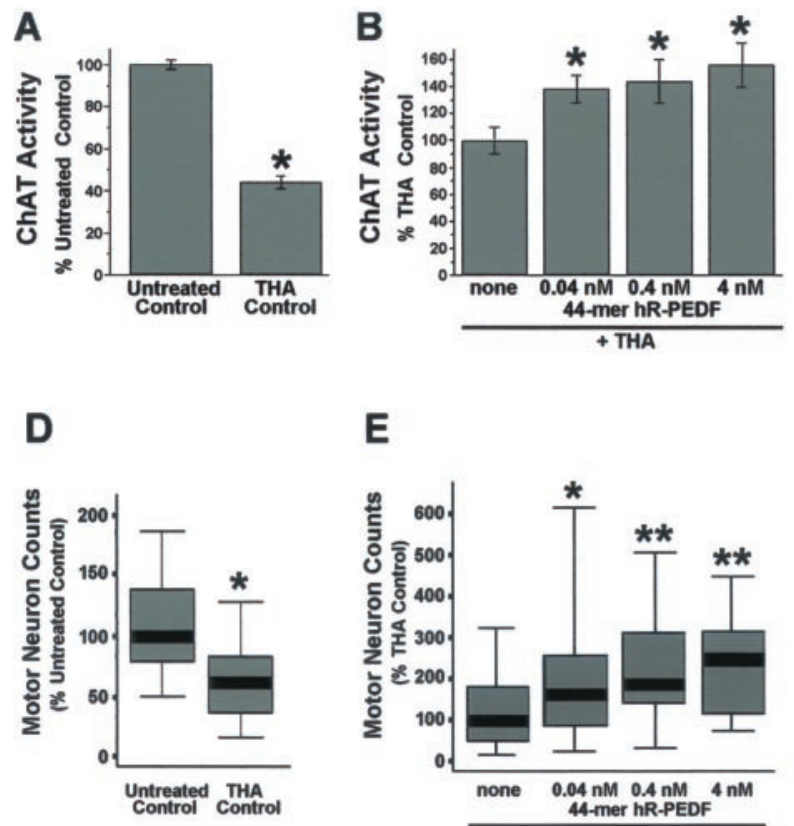

E
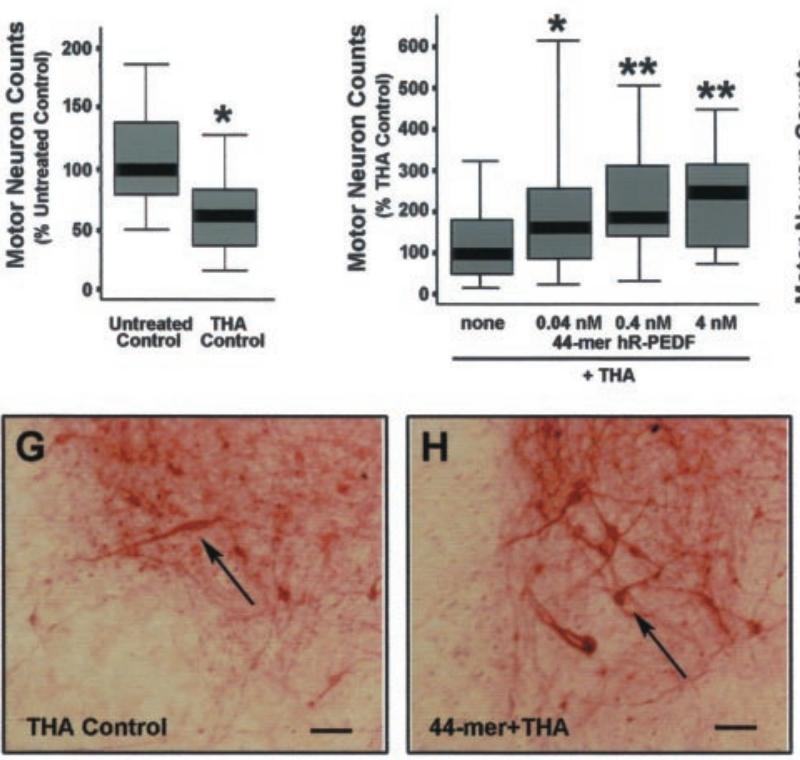

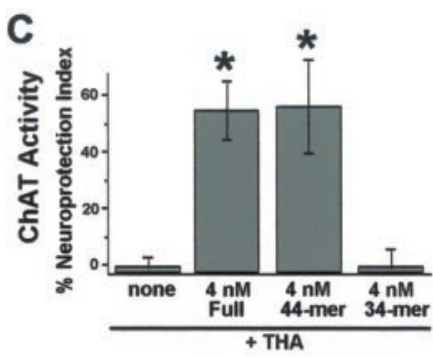

$\mathbf{F}$
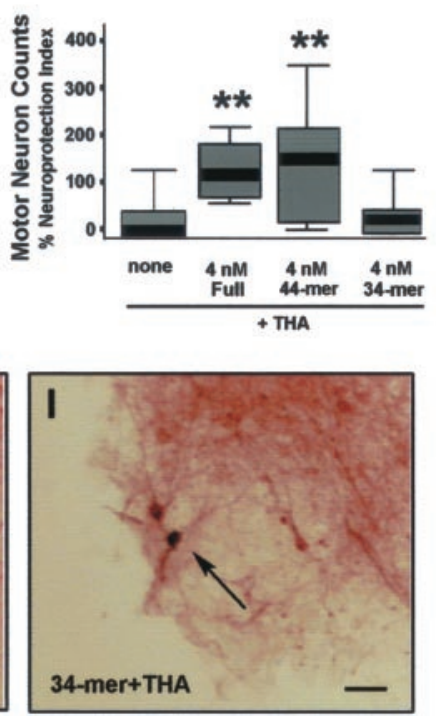

Figure 1. Both the full-length and the 44 mer region of human PEDF protect motor neurons from glutamate-mediated cell death. Motor neuronal protection was assayed by measuring ChAT activity $(A-C)$ and by counting motor neurons in spinal cord slices $(D-F) . A$, Incubation of spinal cord slices with a glutamate transporter inhibitor, THA, on average reduced motor neuron ChAT activity to $44 \%$ of the untreated controls $(* p<$ $1 \times 10^{-24}$ ) after DIV 42-49. $B$, In the same experiments, the 44 mer of PEDF peptide prevented loss of ChAT activity because of chronic glutamate exposure in a doseresponsive manner ( ${ }^{*} p<0.05$ vs THA control). $h R$-PEDF, rhuPEDF. $C$, When we compare the neuroprotection index (percentage of neuroprotection in $B$ minus the THA control) of each peptide at effective stoichiometric concentrations (4 nM shown), the neuroprotective effect of full-length PEDF can be completely accounted for by the 44 mer but not by the 34 mer $\left({ }^{*} p<1 \times 10^{-6}\right.$ vs THA alone). $D-F$, Motor neuron survival was assessed by counting SMI-32 immunostained pyramidal neurons in the ventral horn. $D$, Incubation of spinal cord slices with a glutamate transporter inhibitor, THA, reduced the motor neuron number on average to $60 \%$ of untreated controls $\left({ }^{*} p<1 \times 10^{-4}\right)$ after DIV 42-49. E, The 44 mer of PEDF peptide prevented motor neuron loss in a doseresponsive manner. ${ }^{*} p=0.06 ;{ }^{*} p<0.02$ vs THA controls. $F$, A neuroprotection index was used to compare the action of each PEDF peptide on motor neuron survival at stoichiometrically equivalent $4 \mathrm{~nm}$ doses. In agreement with ChAT assay data, the fulllength PEDF and the 44 mer but not the 34 mer significantly enhanced motor neuron survival. ${ }^{* *} p<0.02$ vs THA control; $n=13-15$ spinal cord slices per experimental group. $G-I$, Immunocytochemistry of organotypic cultures for nonphosphorylated NF-H in neuronal cell bodies and their processes. In cultures exposed to $100 \mu \mathrm{M}$ THA, NF-H immunostaining reveals a severe loss of motor neurons in the ventral horn $(G)$. Concurrent treatment of THA-intoxicated cultures with the 44 mer $(0.4$ nM shown) dramatically and reproducibly preserves motor neurons $(H)$, and the cultures appear similar to those treated with full-length PEDF (data not shown). In contrast, treatment with the 34 mer ( 0.4 nM shown) does not prevent the THA-mediated loss of motor neurons $(I)$, and the cultures appear similar to those treated with THA alone $(G)$. Arrows, SMI-32-positive motor neurons. Scale bars, $100 \mu \mathrm{m}$.

ner (Fig. $3 C-G$ ). The binding pattern appeared the same among the three concentrations $(4,20$, or $50 \mathrm{~nm})$ of the probe used, but the intensity increased proportionally with the probe concentration. Contrary to what we observed with slice cultures, no fluorescein signal was observed in the nucleus of isolated embryonic motor neurons, even with the higher $50 \mathrm{~nm}$ concentration of Fl-PEDF. This may indicate the existence of different PEDF signaling pathways in motor neurons at different developmental stages (e.g., autocrine vs paracrine patterns). The fluorescein signal was virtually absent when motor neurons were incubated with $20 \mathrm{~nm}$ Fl-PEDF plus a 50-fold excess of unlabeled PEDF (data not shown).

\section{Binding analyses of ${ }^{125}$ I-PEDF in spinal cord}

To investigate the presence of the receptors in spinal cord slices used for those biological assays, we performed radioligand binding reactions. Incubation of spinal cord slice cultures with $4 \mathrm{nM}$ ${ }^{125}$ I-PEDF showed a time course of specific binding as early as 15 min that was saturable by $4 \mathrm{hr}$ (Fig. $4 A$ ), implying the existence of PEDF receptors in cells of the spinal cord slices.

To examine the PEDF binding profile to cell-surface receptors on motor neurons, we determined the physicochemical parameters using single-cell cultures of motor neurons. Binding was performed with a given amount of ${ }^{125}$ I-PEDF radioligand and increasing concentrations of unlabeled PEDF. The saturation binding isotherm shows that motor neurons exhibited saturable and specific binding of PEDF (Fig. 4B). Furthermore, the Scatchard data plot of the transformed data demonstrated a single class of binding sites. Nonlinear regression analysis of the binding data with one binding site revealed that motor neurons had $\sim 48,000$ PEDF binding sites per cell, with an apparent dissociation constant $\left(K_{\mathrm{d}}\right)$ of $7.9 \mathrm{~nm}$ (Fig. $4 C$, one representative experiment). Nonlinear regression with two binding sites did not converge in any experiment. The observed $\mathrm{EC}_{50}$ in that experiment was $10 \mathrm{~nm}$. Second and third experiments performed with different batches of motor neuron cultures revealed similar kinetics. Overall mean $K_{\mathrm{d}}$ was $11.4 \mathrm{~nm}$ (range, 2.4-18.9) and mean $B_{\max }$ was 57,440 sites per cell (range, 37,246-76,708).

\section{PEDF increases the survival of rat embryonic motor neurons}

We analyzed the biological responses of our rat motor neuron preparation to PEDF. These experiments were performed in enriched cultures shown to contain $80-85 \%$ motor neurons (Fig. 5). Most surviving cells had a morphology resembling motor neurons (i.e., large, containing at least one long neurite, and a 


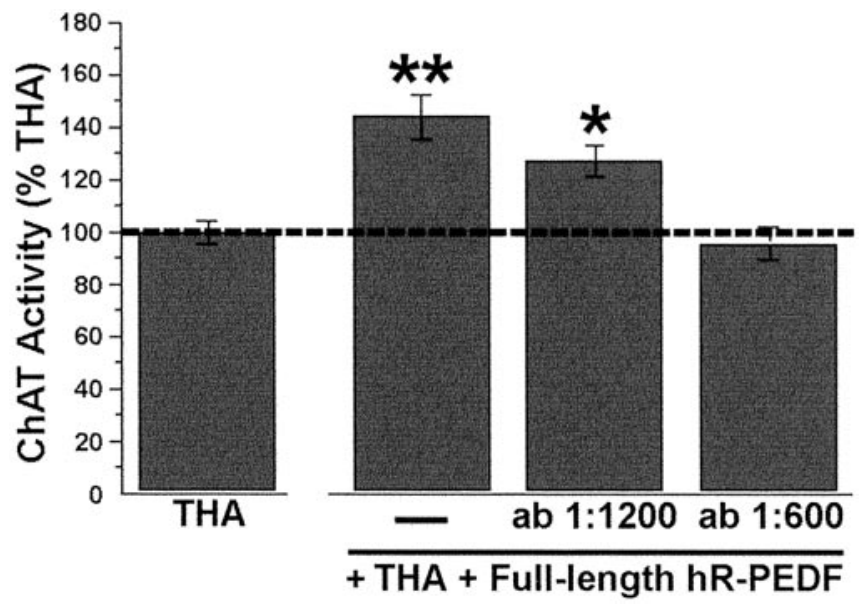

Figure 2. Neuroprotection by rhuPEDF ( $h R-P E D F)$ can be blocked by PEDF antiserum. In spinal cord cultures that were chronically intoxicated with THA, full-length PEDF (4 nM) prevented loss of ChAT activity $\left({ }^{* *} p<1 \times 10^{-4}\right.$ vs THA alone). However, preincubation of PEDF with antiserum $(a b)$ to PEDF at a dilution of 1:1200 resulted in partial neutralization of the neuroprotective effect of PEDF ( ${ }^{*} p=0.04$ vs THA). The blockade of the protection was complete with the antiserum at a 1:600 dilution (i.e., there was no significant difference in ChAT activity between cultures treated with THA plus the antiserum-adsorbed PEDF and those treated with THA alone) ( $p=0.69$ vs THA). ChAT activity in control cultures, with or without THA, was not affected by treatment with PEDF antiserum itself (data not shown). The dashed line indicates the THA control.

typically pyramidal- or bipolar-shaped cell body). Cells that did not fit this set of criteria were not included in the motor neuron counts. Most cells that received motor neuron medium lacking B27 (negative control) degenerated by DIV 4 (Fig. 6 $A$ ). In contrast, significantly more motor neurons survived in the medium containing PEDF (Fig. 6B,D) $\left(p<1 \times 10^{-6}\right.$ vs negative control). Cultures that received B27 consistently had the highest mean numbers of motor neurons (Fig. $6 C)\left(p<1 \times 10^{-15}\right.$ vs negative control). These results indicate that single-cell motor neuron cultures respond to PEDF by increasing their survival. In conjunction with our binding data (shown above), these observations demonstrate that the survival effect of PEDF on motor neurons is attributable to a direct interaction of PEDF with cell-surface receptors.

\section{DISCUSSION}

\section{PEDF as a survival factor for motor neurons and development as a therapy for amyotrophic lateral sclerosis}

We show in this study that highly purified human recombinant PEDF protects motor neurons from chronic glutamate-mediated degeneration. This neuroprotection is blocked by a neutralizing PEDF antiserum, confirming the specificity of PEDF as the neuroprotectant within the recombinant preparation (Fig. 2). In addition, the fact that the neuroprotective activity is completely attributable to a 44 mer peptide from the $\mathrm{N}$ terminal of the 418 aa human PEDF molecule points out that this region spanning amino acid positions 78-121 contains the neuroprotective active site for motor neurons (Fig. 1). Neuroprotective small peptides that can generate or mimic the activities of neurotrophic factors may offer advantages in the development of neuroprotective therapeutics for motor neuron diseases and spinal cord disorders (Gozes, 2001). Theoretically, the simpler the neuroprotective
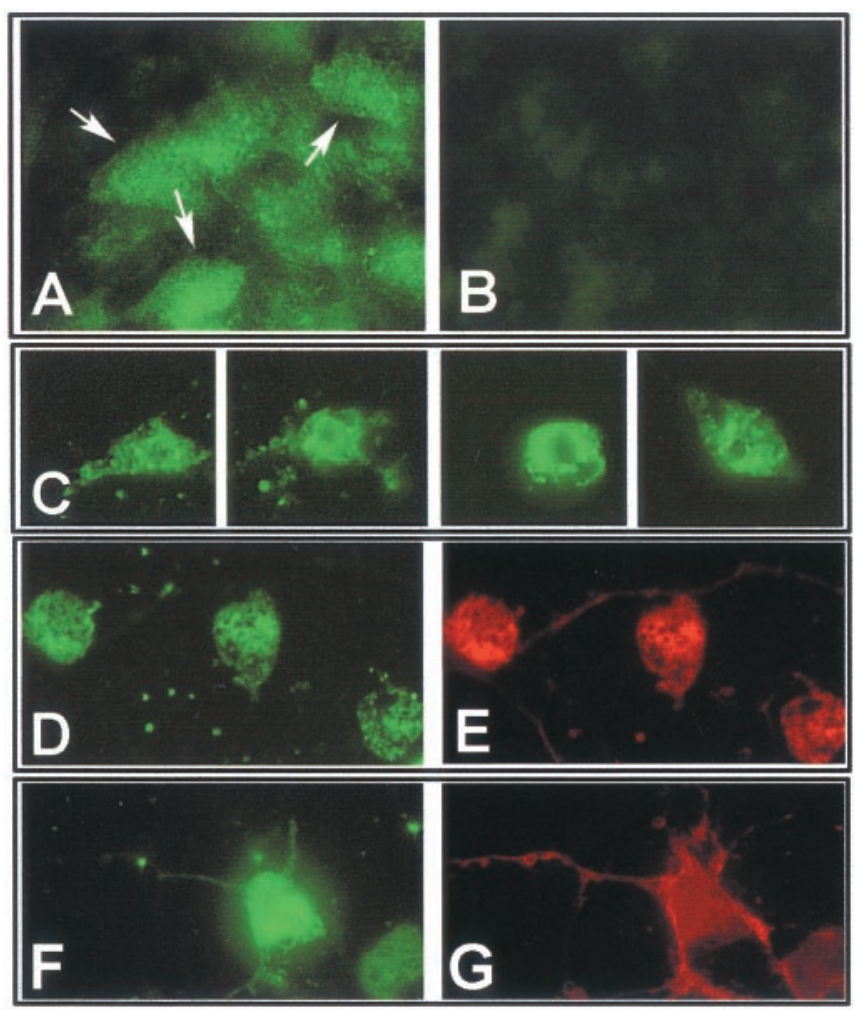

Figure 3. Fl-PEDF binding to the spinal cord. $A$, A field from the ventral gray matter of a $350 \mu \mathrm{m}$ slice that was incubated with $20 \mathrm{nM}$ Fl-PEDF for $1 \mathrm{hr}$. Intense punctate staining on large neurons (arrows) indicates the binding sites for fluorescein molecules that were conjugated to full-length PEDF protein. $B$, A representative field from the ventral gray matter of untreated unlabeled spinal cord showing the background level. $C$, Four examples of isolated motor neurons in single-cell cultures that contained Fl-PEDF (50 nM shown). $D$, Photomicrograph showing isolated motor neurons that contained fluorescein signal. $E$, Same field as in $D$, but taken with a rhodamine filter. Virtually all cells that contained Fl-PEDF binding sites were immunopositive for NF-H. F, $G$, A rare example of isolated motor neurons that contained Fl-PEDF binding sites $(F)$ in both cell body and processes, which were visualized with SMI-32 antibody $(G)$.

molecule, the fewer problems with immunogenicity and CNS access.

The potential neuroprotective effect of PEDF on mature motor neurons has broad implications. Although we have used a slow glutamate-mediated neurodegeneration model here because of its relevance for amyotrophic lateral sclerosis (ALS) (Shaw and Kuncl, 2002), the future value of PEDF as a neuronal protectant is not dependent on which multiple pathophysiologic mechanisms of cell death turn out to be true in ALS. This breadth may be best illustrated by the many model systems in which PEDF has been validated. PEDF reportedly promotes survival from acute glutamate toxicity of cultured neonatal hippocampal neurons (DeCoster et al., 1999) and postnatal CG neurons (Taniwaki et al., 1997). These properties may be more relevant to ischemia and trauma than to neurodegeneration. PEDF also offers protection against apoptotic cell death in embryonic motor neurons and immature (defined as DIV 0-3) CG cells in culture (Araki et al., 1998; Houenou et al., 1999).

It is pertinent to neurodegenerative diseases that PEDF is present throughout development in mammalian species, including humans, in various organs such as muscle, biological fluids such as $\mathrm{CSF}$, and in the CNS (including motor neurons and ependymal 

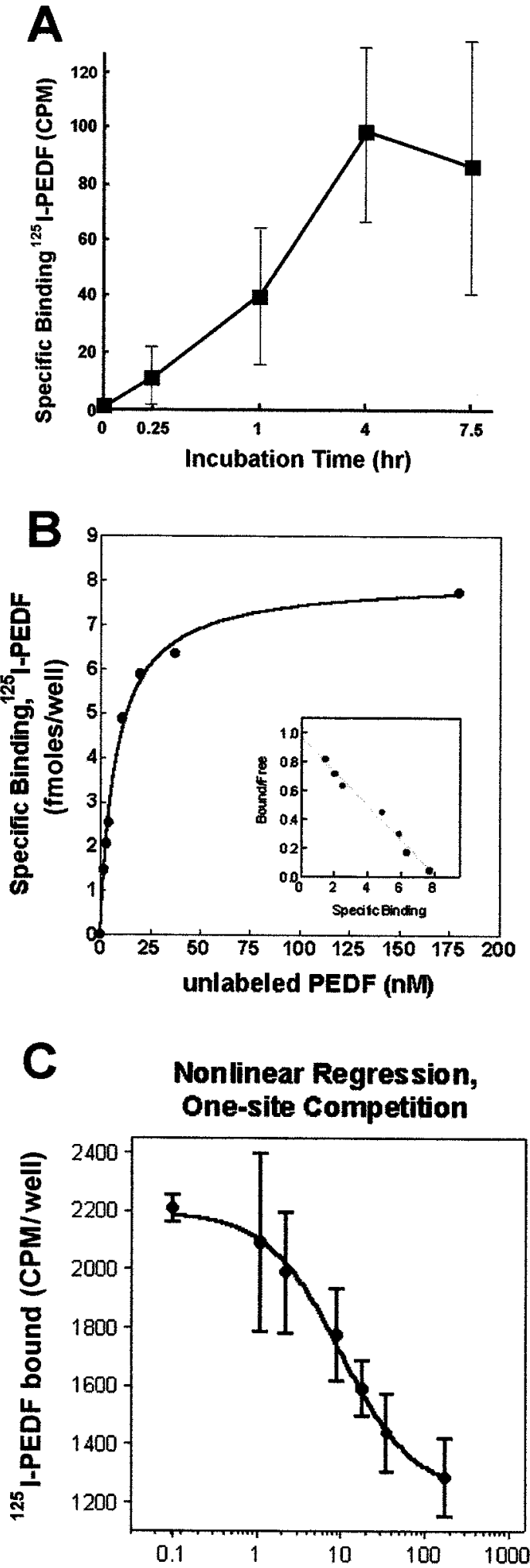

Figure 4. Binding characteristics of ${ }^{125} \mathrm{I}$-PEDF. $A$, Time course of ${ }^{125} \mathrm{I}$ PEDF binding in the spinal cord showing the specific binding after each incubation time. Binding reactions contained spinal cord slices with $4 \mathrm{~nm}$ radioligand. Incubations were at $4^{\circ} \mathrm{C}$ for the indicated amount of time. Each experimental point is the average of triplicates. Bound radioactivity against incubation time is plotted from one representative experiment. $B$, To examine the PEDF binding profile to cell-surface receptors on motor neurons, we determined the physicochemical parameters using single-cell cultures of motor neurons. Binding was performed with a given amount of
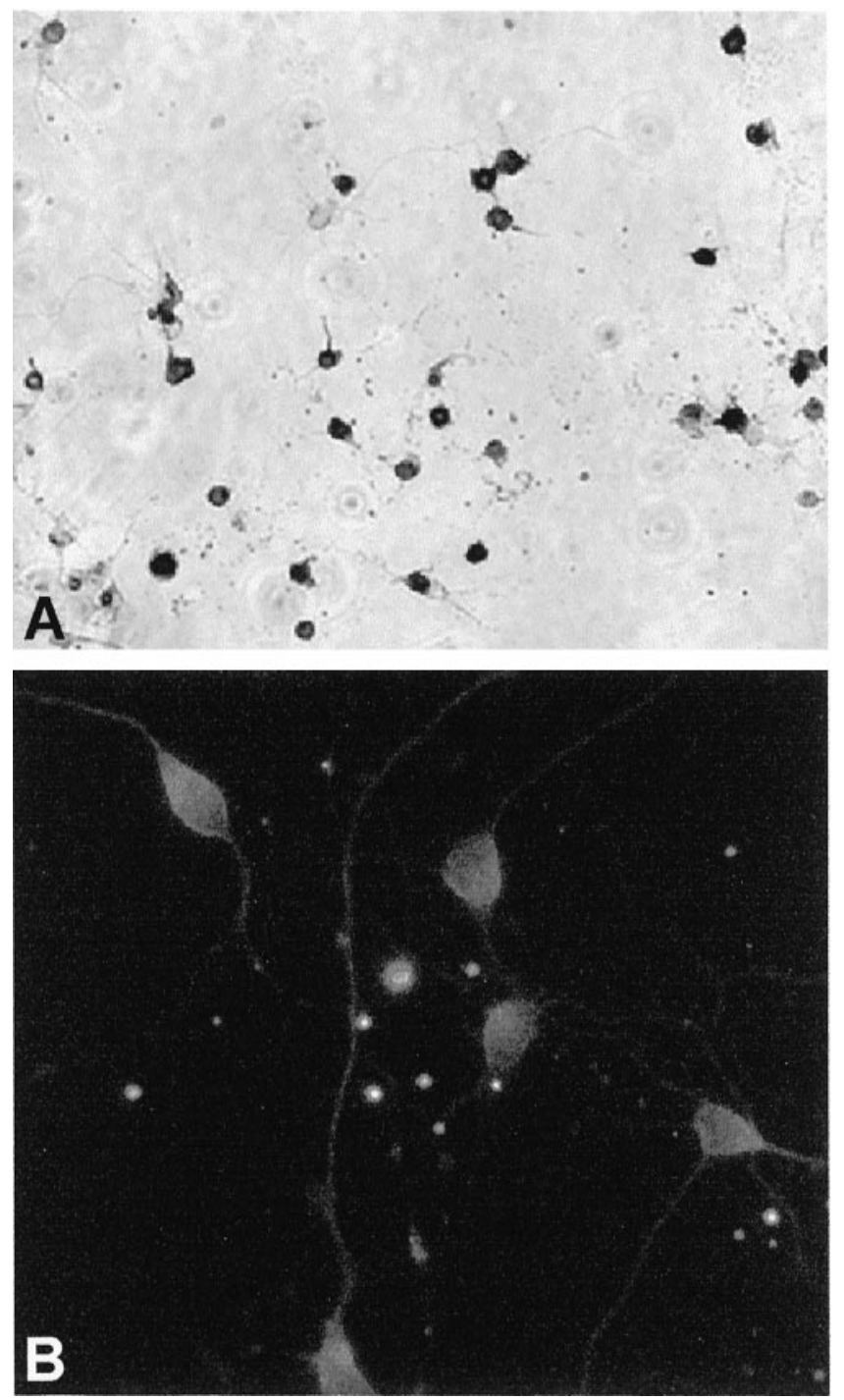

Figure 5. Rat embryonic motor neuron-enriched cultures. A typical microscopic field showing that the vast majority of the cells are immunopositive for islet-1 $(A)$ and with SMI-32 $(B)$. Islet-1 immunostaining was visualized using the VectaABC Kit (Vector Laboratories) and DAB, and SMI-32 immunostaining was visualized with horse anti-mouse Texas Red. The photograph in $A$ was taken with a $20 \times$ objective lens, and the one in $B$ was taken with a $63 \times$ objective lens.

cells in the spinal cord) (Tombran-Tink et al., 1996; Wu and Becerra, 1996; Bilak et al., 1999a). Thus, PEDF may play important roles in the survival and maintenance of various kinds of neurons, including spinal motor neurons, not only during fetal development but also in their neuroprotection against acquired insults in late postnatal life. Reports describing the regulation of PEDF in human disorders are accumulating. We have shown

\footnotetext{
${ }^{125}$ I-PEDF radioligand and increasing concentrations of unlabeled PEDF. The saturation-binding isotherm shows that motor neurons exhibit saturable and specific binding of PEDF to cell-surface receptors (1 representative experiment shown). The Scatchard plot (inset) shows the transformed data. The half-maximal effect of adding unlabeled PEDF $\left(\mathrm{EC}_{50}\right)$ was 10.12 nM. $C$, Nonlinear regression analysis of the binding data with one binding site revealed that motor neurons have $\sim 48,000$ PEDF binding sites per cell with an apparent $K_{\mathrm{d}}$ of $7.9 \mathrm{nM}$.
} 

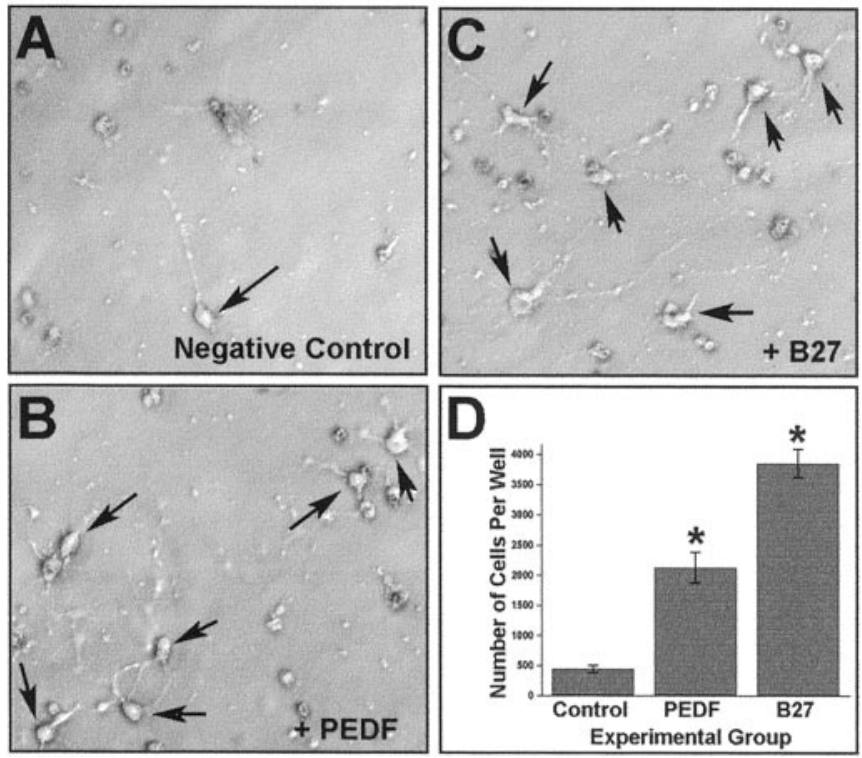

Figure 6. PEDF increases the survival of rat embryonic motor neurons. $A$, Phase micrograph showing DIV 4 culture incubated with medium lacking B27. Many degenerated cells are seen. B, Age-matched culture incubated with the medium supplemented with PEDF, showing many surviving neurons (arrows). C, Age-matched culture that received B27 (as a positive control), showing many surviving motor neurons (arrows). Only cells with neurites were counted as surviving motor neurons in all groups. $n=18$ wells per experimental group. $D$, Summary plot showing mean numbers of motor neurons per well from each experimental group. ${ }^{*} p<$ $1 \times 10^{-6}$ versus negative control group.

recently that PEDF levels are specifically increased in the CSF of individuals with ALS (means, $1.0 \mu \mathrm{g} / \mathrm{ml}$ in disease controls vs 1.3 $\mu \mathrm{g} / \mathrm{ml}$ in ALS; threefold higher in ALS than in controls when normalized to total protein) (Kuncl et al., 2002) (note that 0.2 $\mu \mathrm{g} / \mathrm{ml}$ of full-length PEDF equals $\sim 4 \mathrm{~nm})$. Although the mechanism by which PEDF is elevated in ALS CSF is not clear, we speculate that the elevated levels of PEDF may be an autoprotective reaction in ALS. Ogata et al. (2001) reported recently that in patients with rhegmatogenous retinal detachment, PEDF levels are elevated in the vitreous fluid, where it may act as a neuroprotectant for the detached retina. The neuroprotective potential of PEDF has not yet been studied in animal models of motor neuron degeneration in vivo. However, the safety and efficacy of PEDF have been reported recently using systemic administration of PEDF in a mouse model of ischemia-induced retinopathy (Stellmach et al., 2001). PEDF may preferentially act on "abnormal" or "sick" cells. For example, it protects glutamate-injured motor neurons but does not offer neurotrophic/neuritogenic effects on normal, mature motor neurons (Bilak et al., 1999a). As another example, Stellmach et al. (2001) demonstrated that PEDF prevents angiogenesis by inducing apoptosis in endothelial cells that are presumably on their way to the vitreous, without harming the endothelial cells that are present in already established vessels. These authors speculated that destabilized cells may possibly be "activated" and thus more susceptible to PEDF.

\section{Mechanisms of action for PEDF and intracellular signaling}

The fact that the 44 mer peptide conferred the neuroprotective activity of the PEDF polypeptide first points to mechanisms of action that are independent of the protease inhibition potential of the serpin PEDF. Using two different ligands, Fl-PEDF and
${ }^{125}$ I-PEDF, we demonstrated herein that motor neurons contain specific and high-affinity receptors for PEDF on their cell surfaces. The inhibition of the neuroprotective activity of PEDF by monospecific antiserum to PEDF implies interactions of PEDF and the antiserum, preventing the binding of PEDF on the surface of motor neurons. Thus, binding to cell-surface receptors in motor neurons is considered the first step in the biological effect of PEDF. That the binding affinity of PEDF for receptors in rat motor neurons $\left(K_{\mathrm{d}}\right.$ range, $\left.2.4-18.9 \mathrm{~nm}\right)$ resembles that in human retinoblastoma Y-79 cells ( $3 \mathrm{nM})$, rat CG cells $(4.5 \mathrm{nM})$, and bovine retinal cells $(6.5 \mathrm{~nm})$ suggests a homologous protein for the PEDF receptor in these neuronal systems (Fig. 4) (Alberdi et al., 1999; Aymerich et al., 2001a). The distribution of PEDF receptors on spinal cord motor neurons is in agreement with the protective effects of PEDF on motor neurons (Figs. 1, 6) (Bilak et al., 1999a; Houenou et al., 1999), implying that these are functional receptors that, on interaction with the ligand, PEDF, can directly trigger a signaling cascade for neuroprotection.

The mechanisms for the neuroprotective actions of PEDF and signaling pathways it may activate in motor neurons are not known. Comparison with other serpins that have an effect on motor neurons indicated that the mechanisms of action of PEDF are unique. Some serpins can function as a neurotrophic/neuroprotective factor in the CNS. For example, an inhibitory serpin protease, nexin-I, protects embryonic and mature motor neurons from programmed and axotomy-induced cell death, respectively, and enhances their neurite outgrowth (Brenneman et al., 1987; Zurn et al., 1988; Houenou et al., 1995) by inhibiting its target protease thrombin (Festoff et al., 1996). In addition, it has been proposed that an imbalance of serine proteases and their cognate serpins may have a role in motor neuron degeneration in the pathogenesis of ALS (Chou et al., 1998). We have shown that PEDF is elevated in the CSF of patients with ALS. However, whether PEDF is involved in the pathogenesis of ALS is not clear.

Second, PEDF may protect neurons by altering intracellular calcium homeostasis. In an acute glutamate neurotoxicity model, PEDF was reported to protect CG neurons by reducing the plateau level of intracellular calcium rather than by reducing the initial rise in intracellular calcium (Taniwaki et al., 1997). The authors speculated that PEDF may regulate calcium-reducing mechanisms, such as calcium pumps, $\mathrm{Na}^{+}-\mathrm{Ca}^{2+}$ exchanger, or calcium-binding proteins. We have shown that other motor neuron protectants, namely IGF-I and glial cell line-derived neurotrophic factor (GDNF), can increase the expression of calbindin and parvalbumin, two of the calcium-binding proteins, in glutamate-injured spinal cord (Bilak et al., 2000). Whether PEDF can protect mature motor neurons by a similar calcium-mediated mechanism has not yet been tested.

Third, PEDF could protect neurons against glutamate excitotoxicity through alteration of the synthesis and/or release of other factors such as neurotrophins and neurotransmitters. It has been demonstrated recently that PEDF directly protects isolated postnatal CG neurons from glutamate-mediated necrotic cell death by inducing the activity of the transcription factor nuclear factor- $\kappa \mathrm{B}$ (Yabe et al., 2001). In the same culture system, PEDF also upregulated the mRNA for NGF, BDNF, and GDNF but did not regulate the anti-apoptotic genes $\mathrm{Bcl}-2, \mathrm{Bcl}-\mathrm{x}$, and $\mathrm{Mn}$-SOD.

Finally, neuroprotective effects of PEDF on motor neurons may also be indirectly regulated by glia. PEDF increases the metabolic activities of isolated microglia and inhibits the proliferation of both microglia and astrocytes; thus, it may have an 
important role in the regulation of glial functions (Sugita et al., 1997). This finding also suggests that receptors for PEDF are also present on microglia. In agreement with this hypothesis, we have observed the presence of Fl-PEDF binding sites on small nonneuronal cells in our spinal cord slices (data not shown). If PEDF receptor activation on glia signaled the increased expression or activity of the glial glutamate transporter, that could be a possible indirect mechanism of its motor neuron protective effect.

Our data also provide information on the structure-function relationships of the neuroprotective activity of PEDF. A previous report demonstrated that the 44 mer peptide is neurotrophically active and that it contains the receptor-binding region of PEDF in retinoblastoma Y-79 cells (Alberdi et al., 1999). This region of PEDF is distinct and nonoverlapping with a glycosaminoglycanbinding region in the folded structure of the PEDF protein (Alberdi et al., 1998). To our knowledge, the present study is the first report showing that this small PEDF peptide contains the structural determinants for motor neuron survival. In summary, this study indicates that a small proximal peptide fragment of the PEDF molecule, independent of protease inhibition, could be engineered to contain all of its motor neuron protective activity, and that the neuroprotective action is likely to be mediated directly on motor neurons via a single class of PEDF receptor. These data should provide a strong preclinical rationale for the use of PEDF in therapy for ALS. Future studies will include molecular characterization of these receptors and elucidation of the intracellular signal transduction events that PEDF ligandreceptor interactions may activate to exert neurotrophic and neuroprotective actions on motor neurons. However, we have established that the first step in those biological events is the binding to cell-surface receptors on motor neurons.

\section{REFERENCES}

Alberdi E, Hyde CC, Becerra SP (1998) PEDF binds to glycosaminoglycans: analysis of binding site. Biochemistry 37:10643-10652.

Alberdi E, Aymerich MS, Becerra SP (1999) Binding of pigment epithelium-derived factor (PEDF) to retinoblastoma cells and cerebellar granule neurons: evidence for a PEDF receptor. J Biol Chem 274:31605-31612.

Araki T, Taniwaki T, Becerra SP, Chader GJ, Schwartz JP (1998) Pigment epithelium-derived factor (PEDF) differentially protects immature but not mature cerebellar granule cells against apoptotic cell death. J Neurosci Res 53:7-15.

Aymerich MS, Alberdi EM, Martinez A, Becerra SP (2001a) Evidence for pigment epithelium-derived factor receptors in the neural retina. Invest Ophthalmol Vis Sci 42:3287-3293.

Aymerich MS, Martinez A, Becerra SP (2001b) Characterization and localization of pigment epithelium-derived factor binding sites in the bovine retina. In: New insights in retinal degenerative diseases (Anderson RE, LaVail MM, Hollyfield JG, eds), pp 1-7. New York: Kluwer Academic/Plenum.

Becerra SP (1997) Structure-function studies on PEDF: a noninhibitory serpin with neurotrophic activity. Adv Exp Med Biol 425:223-237.

Becerra SP, Palmer I, Kumar A, Steele F, Shiloach J, Notario V, Chader GJ (1993) Overexpression of fetal human pigment epithelium-derived factor in Escherichia coli: a functionally active neurotrophic factor. J Biol Chem 268:23148-23156.

Becerra SP, Sagasti A, Spinella A, Spinella P, Notario V (1995) Pigment epithelium-derived factor behaves like a noninhibitory serpin. J Biol Chem 270:25992-25999.

Bilak MM, Corse MA, Bilak RS, Lehar M, Tombran-Tink J, Kuncl RW (1999a) Pigment epithelium-derived factor (PEDF) protects motor neurons from chronic glutamate-mediated neurodegeneration. J Neuropathol Exp Neurol 58:719-728.

Bilak MM, Shifrin DA, Corse AM, Bilak SR, Kuncl RW (1999b) Neuroprotective utility and neurotrophic action of neurturin in postnatal motor neurons: comparison with GDNF and persephin. Mol Cell Neurosci 13:326-336.

Bilak MM, Moss BH, Kuncl RW (2000) Insulin-like growth factor I (IGF-I) and glial cell line-derived neurotrophic factor (GDNF) increases calbindin expression in glutamate-injured ventral horn neurons. Mol Biol Cell 11:412a.
Bilak MM, Corse AM, Kuncl RW (2001) Additivity and potentiation of IGF-I and GDNF in the complete rescue of postnatal motor neurons. Amyotroph Lateral Scler Other Motor Neuron Disord 2:83-91.

Brenneman DE, Phillips TM, Festoff BW, Gozes I (1987) Identity of neurotrophic molecules released from astroglia by vasoactive intestinal peptide. Ann NY Acad Sci 814:167-173.

Cao W, Tombran-Tink J, Elias R, Sezate S, Mrazek D, McGinnis JF (2001) In vivo protection of photoreceptors from light damage by pigment epithelium-derived factor. Invest Ophthalmol Vis Sci 42:1646-1652.

Cayouette M, Smith SB, Becerra SP, Gravel C (1999) Pigment epithelium-derived factor delays the death of photoreceptors in mouse models of inherited retinal degenerations. Neurobiol Dis 6:523-532.

Chou SM, Taniguchi A, Wang HS, Festoff BW (1998) Serpin=serine protease-like complexes within neurofilament conglomerates of motoneurons in amyotrophic lateral sclerosis. J Neurol Sci 160 [Suppl 1]:S73-S79.

Corse AM, Bilak MM, Bilak SR, Lehar M, Rothstein JD, Kuncl RW (1999) Preclinical testing of neuroprotective neurotrophic factors in a model of chronic motor neuron degeneration. Neurobiol Dis 6:335-346.

Dawson DW, Volpert OV, Gillis P, Crawford SE, Xu H, Benedict W, Bouck NP (1999) Pigment epithelium-derived factor: a potent inhibitor of angiogenesis. Science 285:245-248.

DeCoster MA, Schabelman E, Tombran-Tink J, Bazan NG (1999) Neuroprotection by pigment epithelial-derived factor against glutamate toxicity in developing primary hippocampal neurons. J Neurosci Res $56: 604-610$.

Doolittle RF (1983) Angiotensinogen is related to the antitrypsinantithrombin-ovalbumin family. Science 222:417-419.

Ericson J, Thor S, Edlund T, Jessell TM, Yamada T (1992) Early stages of motor neuron differentiation revealed by expression of homeobox gene Islet-1. Science 256:1555-1560.

Festoff BW, Nelson PG, Brenneman DE (1996) Prevention of activitydependent neuronal death: vasoactive polypeptide stimulates astrocytes to secrete the thrombin-inhibiting neurotrophic serpin, protease nexin I. J Neurobiol 30:255-266.

Gao G, Li Y, Zhang D, Gee S, Crosson C, Ma J (2001) Unbalanced expression of VEGF and PEDF in ischemia-induced retinal neovascularization. FEBS Lett 489:270-276.

Gozes I (2001) Neuroprotective peptide drug delivery and development: potential new therapeutics. Trends Neurosci 24:700-705.

Houenou LJ, Turner PL, Li L, Oppenheim RW, Festoff BW (1995) A serine protease inhibitor, protease nexin I, rescues motoneurons from naturally occurring and axotomy-induced cell death. Proc Natl Acad Sci USA 92:895-899.

Houenou LJ, D'Costa AP, Li L, Turgeon VL, Enyadike C, Alberdi E, Becerra SP (1999) Pigment epithelium-derived factor promotes the survival and differentiation of developing spinal motor neurons. J Comp Neurol 412:506-514.

Jablonski MM, Tombran-Tink J, Mrazek DA, Iannaccone A (2000) Pigment epithelium-derived factor supports normal development of photoreceptor neurons and opsin expression after retinal pigment epithelium removal. J Neurosci 20:7149-7157.

Kuncl RW, Bilak MM, Bilak SR, Corse AM, Royal W, Becerra SP (2002) Pigment epithelium-derived factor (PEDF) is elevated in cerebrospinal fluid (CSF) of patients with amyotrophic lateral sclerosis (ALS). J Neurochem 81:178-184.

Mori K, Duh E, Gehlbach P, Ando A, Takahashi K, Pearlman J, Yang HS, Zack DJ, Ettyreddy D, Brough DE, Wei LL, Campochiaro PA (2001) Pigment epithelium-derived factor inhibits retinal and choroidal neovascularization. J Cell Physiol 188:253-263.

Nomura T, Yabe T, Mochizuki H, Reiser J, Becerra SP, Schwartz JP (2001) Survival effects of pigment epithelium-derived factor expressed by a lentiviral vector in rat cerebellar granule cells. Dev Neurosci 23:145-152.

Ogata N, Tombran-Tink J, Nishikawa M, Nishimura T, Mitsuma Y, Sakamoto T, Matsumura M (2001) Pigment epithelium-derived factor in the vitreous is low in diabetic retinopathy and high in rhegmatogenous retinal detachment. Am J Ophthalmol 132:378-382.

Rothstein JD, Jin L, Dykes-Hoberg M, Kuncl RW (1993) Chronic inhibition of glutamate uptake produces a model of slow neurotoxicity. Proc Natl Acad Sci USA 90:6591-6595.

Shaw PJ, Kuncl RW (2002) Current concepts in the pathogenesis of amyotrophic lateral sclerosis. In: Motor neuron diseases (Major problems in neurology series) (Kuncl RW, ed), pp 37-74. London: Saunders.

Steele FR, Chader GJ, Johnson LV, Tombran-Tink J (1993) Pigment epithelium-derived factor (PEDF): neurotrophic activity and identification as a unique member of the serine protease inhibitor (SERPIN) gene family. Proc Natl Acad Sci USA 90:1526-1530.

Stellmach V, Crawford SE, Zhou W, Bouck N (2001) Prevention of ischemia-induced retinopathy by the natural ocular anti-angiogenic agent pigment epithelium-derived factor. Proc Natl Acad Sci USA 98:2593-2597.

Stratikos E, Alberdi E, Gettins PG, Becerra SP (1996) Recombinant human pigment epithelium-derived factor (PEDF): characterization of 
PEDF overexpressed and secreted by eukaryotic cells. Protein Sci 5:2575-2582.

Sugita Y, Becerra SP, Chader GJ, Schwartz JP (1997) Pigment epithelium-derived factor (PEDF) has direct effects on the metabolism and proliferation of microglia and indirect effects on astrocytes. J Neurosci Res 49:710-718.

Taniwaki T, Becerra SP, Chader GJ, Schwartz JP (1995) Pigment epithelium-derived factor is a survival factor for cerebellar granule cells in culture. J Neurochem 64:2509-2517.

Taniwaki T, Hirashima N, Becerra SP, Chader GJ, Etcheberrigaray R, Schwartz JP (1997) Pigment epithelium-derived factor protects cultured cerebellar granule cells against glutamate-induced neurotoxicity. J Neurochem 68:26-32.

Tombran-Tink J, Mazuruk K, Rodriguez IR, Chung D, Linker T, Englander E, Chader GJ (1996) Organization, evolutionary conservation, expression and unusual Alu density of the human gene for pigment epithelium-derived factor, a unique neurotrophic serpin. Mol Vis 2:1-12.

Tsang YM, Chiong F, Kuznetsov D, Kasarskis E, Geula C (2000) Motor neurons are rich in non-phosphorylated neurofilaments: cross-species comparison and alterations in ALS. Brain Res 861:45-58.

Wagner SL, Geddes JW, Cotman CW, Lau AL, Gurwitz D, Isackson PJ, Cunningham DD (1989) Protease nexin-1, an antithrombin with neurite outgrowth activity, is reduced in Alzheimer disease. Proc Natl Acad Sci USA 86:8284-8288.

Wu YQ, Becerra SP (1996) Proteolytic activity directed toward pigment epithelium-derived factor in vitreous of bovine eyes: implications of proteolytic processing. Invest Ophthalmol Vis Sci 37:1984-1993.

Wu Y-Q, Notario V, Chader GJ, Becerra SP (1995) Identification of pigment epithelium-derived factor in the interphotoreceptor matrix of bovine eyes. Protein Exp Purif 6:447-456.

Yabe T, Wilson D, Schwartz JP (2001) NF $\mathrm{B}$ activation is required for the neuroprotective effects of pigment epithelium-derived factor (PEDF) on cerebellar granule neurons. J Biol Chem 276:43313-43319.

Zurn AD, Nick H, Monard D (1988) A glia-derived nexin promotes neurite outgrowth in cultured chick sympathetic neurons. Dev Neurosci $10: 17-24$ 